\title{
A Depression Network of Functionally Connected Regions Discovered via Multi-Attribute Canonical Correlation Graphs
}

\author{
Jian Kang ${ }^{1 *}$, F. DuBois Bowman ${ }^{2}$, Helen Mayberg ${ }^{3}$ and Han Liu $^{4}$ \\ ${ }^{1}$ Department of Biostatistics, University of Michigan \\ 2 Department of Biostatistics, Columbia University \\ ${ }^{3}$ Department of Psychiatry and Behavioral Sciences, Emory University \\ ${ }^{4}$ Department of Financial Engineering and Operational Research, Princeton University
}

\begin{abstract}
To establish brain network properties associated with major depressive disorder (MDD) using resting-state functional magnetic resonance imaging (RsfMRI) data, we develop a multi-attribute graph model to construct a regionlevel functional connectivity network that uses all voxel level information. For each region pair, we define the strength of the connectivity as the kernel canonical correlation coefficient between voxels in the two regions; and we develop a permutation test to assess the statistical significance. We also construct a network based classifier for making predictions on the risk of MDD. We apply our method to Rs-fMRI data from 20 MDD patients and 20 healthy control subjects in the Predictors of Remission in Depression to Individual and Combined Treatments (PReDICT) study. Using this method, MDD patients can be distinguished from healthy control subjects based on significant differences in the strength of regional connectivity. We also demonstrate the performance of the proposed method using simulation studies.
\end{abstract}

*To whom correspondence should be addressed: Jian Kang; Email: jiankang@umich.edu; Phone: (734)-678-1267; Fax: 734-763-4004 


\section{Introduction}

Major depressive disorder (MDD) is a common mental illness characterized by an all-encompassing low mood accompanied by low self-esteem. An estimated $16 \%$, or 32 to 35 million, US residents are expected to develop MDD at some point during their lifetime (Kessler et al. 2003). Numerous alterations in localized brain activity for MDD patients have been reported as well as changes in the associations between areas from a small set of defined brain regions. Increased metabolic or neural activity in the amygdala has been observed in depressed patients, often associated with emotional information processing, using both PET and fMRI (Abercrombie et al. 1998, Drevets 1999, Sheline et al. 2001, Siegle et al. 2002). Moreover, alterations in neural activity of the subgenual cingulate has been consistently reported in MDD (Ochsner et al. 2002, Mayberg 1997, Drevets 2000), along with decreased prefrontal cortical activity (Baxter et al. 1989).

There has been relatively less work establishing network properties associated with MDD, and previous findings typically focus on connectivity between two or a relatively small number of predefined depression related regions. For example, Siegle et al. (2007) determined decreased functional connectivity between dorsolateral prefrontal activity and amygdala activity, and similarly O‘Reardon et al. (2007) assessed prefrontal-amygdala interactions. Mayberg et al. (1999; 2005) found dysfunctional interactions among specific cortical and limbic brain areas, in particular discovering significant interactions between subregions of frontal cortex and cingulate gyrus. Also, Mayberg and others have extensively studied defined brain networks of depression-related regions including DLPFC, rostral and posterior cingulate gyri, the orbitofrontal cortex, the insula, the subgenual cingulate gyrus, and the hypothalamus (Mayberg 1997; 2003a;b, Seminowicz et al. 2004, James et al. 2009, Craddock et al. 2009). Despite these and other suggested depression-related neuroimaging find- 
ings, there have been few whole-brain network studies of MDD, and there is limited methodology to accommodate voxel-level analyses for a large number of voxels.

A number of statistical methods have been proposed to study functional brain networks, such as independent component analysis (ICA) (Calhoun et al. 2003, Guo 2011). ICA introduces a few latent components that are associated with different brain regions/voxels, and each component is referred to as a clique in the network. The brain regions/voxels within each clique are assumed to be strongly connected, while regions between cliques are weakly connected or not connected. This strong assumption restricts the topological structure of the network, resulting in difficulty in interpretation for many applications. Another difficulty in ICA is how to appropriately choose the number of components. Although a few methods have been proposed, the performance is not always reliable in practice. Also, not every identified independent component is biologically meaningful and easy to interpret.

Many functional connectivity analysis methods have been proposed based on correlation or partial correlation analysis (Sun et al. 2004, Supekar et al. 2008). However, there are many challenges in estimating correlation networks for the whole brain at the voxel-level. A typical fMRI image involves around 300,000 brain voxels, which implies that there are more than 40 billion voxel pairs for a single whole brain connectivity analysis. This ultra-high dimensionality leads to extremely high computational demands. To reduce the computational load, a common method is to pick a set of seed voxels and compute the association between each seed voxel and all of the remaining voxels. However, this approach excludes a vast number of voxels and can be sensitive to the exact location of the seed. Another strategy is to conduct regionlevel analyses where regions are defined by an existing anatomical brain parcellation, such as the 116 regions produced by the automated anatomical labeling (AAL) system (Tzourio-Mazoyer et al. 2002). A region-based analysis often proceeds by av- 
eraging the temporal profiles across all voxels within a region and then computing statistics to quantify between-region associations. This approach does not leverage all of the voxel-level data to construct the network and does not take into account the heterogeneity of voxels within regions, which may lead to bias in estimating the functional connectivity between regions especially when there are a large number of voxels in the regions.

We develop a multi-attribute graph model to construct a region-level functional connectivity network that uses all voxel level information. Specifically, we consider a multi-attribute graph $G=(V, E)$, where $V$ includes $p$ nodes and each node $v_{i} \in V$ has $n_{i}$ attributes. Each $e(i, j) \in E$ represents strong association in the multiple attributes between nodes $i$ and $j$. For region-level functional connectivity analysis, the anatomical region of interest (ROI) is considered as the node, and the multiple attributes within each node are the voxel-level resting state fMRI signals, since each region contains hundreds or thousands of voxels. All the voxel-specific fMRI signals contribute to the functional connectivity between any two regions. Thus, the regionlevel functional connectivity network is regarded as a multi-attribute graph.

One important question is what is an appropriate measure to characterize the strength of the functional connectivity? An alternative to averaging all voxels within regions is to compute the voxel wise cross correlations between the two regions, and then use a summary statistic of those correlations to characterize the association. The summary statistics could be a maximum, mean or median. The main disadvantage of this approach is the huge computational cost. The number of voxel pairs between two regions can reach or even exceed 100,000. Also, some second-moment summary statistics ignore the dependence between the correlations, which may lead to a higher false positive rate in network estimation.

We consider a more appealing association measure: canonical correlation, which 
is a classical tool in multivariate statistics that has been applied in imaging analysis (Friman et al. 2001, Nandy and Cordes 2004). For each region pair, the strength of the connectivity is defined by the canonical correlation coefficient (CCC) or the kernel canonical correlation coefficient $(\mathrm{kCCC})$ between the voxels in the two regions. The statistical significance is assessed by a permutation test. The CCC and kCCC, respectively, characterize the linear dependence and nonlinear dependence in functional connectivity between the voxels in the two regions. With multiple testing corrections, both subject-specific and group-level brain networks are constructed. Our approach accounts for the variation and spatial correlation of voxels within regions. Key advantages of our approach include: 1) it is able to perform whole brain analysis and requires only moderate computing costs; 2) it produces reliable estimates with small sample sizes, which can be even smaller than the number of brain regions considered in the model; 3) it can be used for both single subject connectivity analysis and also group level analysis; 4) it does not require strong assumptions about the probability distributions generating the data; and 5) combining with the support vector machine (SVM) method, it can construct a classifier for making prediction on the disease risk.

Our work is motivated by an analysis of resting-state functional magnetic resonance imaging (Rs-fMRI) data consisting of 20 MDD patients and 20 healthy control (HC) subjects matched by age and gender participating in a depression treatment biomarker study (Dunlop et al. 2012). The Rs-fMRI data enables the assessment of functional connectivity calculated based on the temporal coherence between distinct neural processing units, characterizing a functional brain network. Our goal is to construct resting state functional connectivity networks for MDD patients and for HC subjects, respectively; and examine difference between the two groups of networks.

The rest of the article is organized as follows: in Section 2, we introduce the multi-attribute graph for single subject analysis and construct a permutation test to 
make inference on the subject specific brain network. Also, we propose a method to estimate a group level network. In Section 3, we apply our multi-attribute graph model to analyze resting state fMRI data from MDD patients and HC subjects, examining differences in the group networks. We also perform network based SVM classification and demonstrate high accuracy. In Section 4, we conduct simulation studies to evaluate the performance and properties of the proposed method, and conclude in Section 5 with a brief discussion.

\section{Method}

We propose a multi-attribute network graph model to determine region-level brain functional connectivity using information from all the regional voxels, and we introduce a kernel canonical correlation coefficient $(\mathrm{kCCC})$ as an association measure between the multi-attribute nodes. For ease of exposition, we begin with the single subject case and then discuss extensions for group-level estimation.

\subsection{Brain connectivity analysis for a single subject}

Let $T$ denote the number of fMRI scans. Denote by $x_{i k t}$ the observed fMRI signal at voxel $k$ in region $i$ at time $t$, for $i=1, \ldots, p, k=1, \ldots, n_{i}$ and $t=1, \ldots, T$. Write $\mathbf{x}_{i t}=\left(x_{i 1 t}, \ldots, x_{i n_{i} t}\right)^{\prime}$ and $\mathbf{X}_{i}=\left(\mathbf{x}_{i 1}, \ldots, \mathbf{x}_{i T}\right)^{\prime}$ of dimension $T \times n_{i}$. Suppose the temporal correlation of fMRI signals has been removed via a pre-whitening procedure using an autoregressive model. Thus, we assume that $\mathbf{x}_{i 1}, \ldots \mathbf{x}_{i T}$ are independent and identically distributed. Also, $\mathbf{X}_{i}$ is assumed to be centered and scaled. We propose to use the canonical correlation $\rho_{c}(i, j)$ to measure the brain connectivity between regions $i$ and $j$. The canonical correlation between $\mathbf{x}_{i t}$ and $\mathbf{x}_{j t}$ is defined as the maximum of the correlation between two linear combinations $\boldsymbol{w}_{i}^{\prime} \mathbf{x}_{i t}$ and $\boldsymbol{w}_{j}^{\prime} \mathbf{x}_{j t}$ with 
respect to the vectors of weights $\boldsymbol{w}_{i} \in \mathbb{R}^{n_{i}}$ and $\boldsymbol{w}_{j} \in \mathbb{R}^{n_{j}}$ :

$$
\rho_{c}(i, j)=\max _{\boldsymbol{w}_{i} \in \mathbb{R}^{n_{i}}, \boldsymbol{w}_{j} \in \mathbb{R}^{n_{j}}} \operatorname{corr}\left(\boldsymbol{w}_{i} \mathbf{x}_{i t}, \boldsymbol{w}_{j} \mathbf{x}_{j t}\right) .
$$

To obtain an estimate of $\rho_{c}(i, j)$ given observed data $\mathbf{x}_{i}$, we adopt an eigenvalue formulation. Then (1) is equivalent to

$$
\begin{aligned}
& \hat{\rho}_{c}(i, j)=\max _{\boldsymbol{w}_{i} \in \mathbb{R}^{n_{i}}, \boldsymbol{w}_{j} \in \mathbb{R}^{n_{j}}} \boldsymbol{w}_{i}^{\prime} \mathbf{X}_{i}^{\prime} \mathbf{X}_{j} \boldsymbol{w}_{j}, \\
& \text { subject to } \quad \boldsymbol{w}_{i}^{\prime} \mathbf{X}_{i}^{\prime} \mathbf{X}_{i} \boldsymbol{w}_{i} \leq 1, \quad \boldsymbol{w}_{j}^{\prime} \mathbf{X}_{j}^{\prime} \mathbf{X}_{j} \boldsymbol{w}_{j} \leq 1
\end{aligned}
$$

where the weights $\boldsymbol{w}_{i}$ and $\boldsymbol{w}_{j}$ can be obtained by solving the eigenvalue equations:

$$
\left\{\begin{array}{l}
\left(\mathbf{X}_{j}^{\prime} \mathbf{X}_{j}\right)^{-1} \mathbf{X}_{j}^{\prime} \mathbf{X}_{i}\left(\mathbf{X}_{i}^{\prime} \mathbf{X}_{i}\right)^{-1} \mathbf{X}_{i}^{\prime} \mathbf{X}_{j} \boldsymbol{w}_{j}=\lambda^{2} \boldsymbol{w}_{j} \\
\left(\mathbf{X}_{i}^{\prime} \mathbf{X}_{i}^{\prime}\right)^{-1} \mathbf{X}_{i}^{\prime} \mathbf{X}_{j}\left(\mathbf{X}_{j}^{\prime} \mathbf{X}_{j}\right)^{-1} \mathbf{X}_{j}^{\prime} \mathbf{X}_{i} \boldsymbol{w}_{i}=\lambda^{2} \boldsymbol{w}_{i}
\end{array}\right.
$$

When $\max \left\{n_{i}, n_{j}\right\} \leq T$, we can solve (3) directly as suggested by Katenka and Kolaczyk (2011). When $\max \left\{n_{i}, n_{j}\right\}>T$, a very common case in fMRI data analysis, the solution of (3) is not unique. The kernel CCA can be used to solve the case that $\max \left\{n_{i}, n_{j}\right\}>T$. Let $\phi_{n}: \mathbb{R}^{n} \rightarrow \mathcal{F}_{\mathbb{R}^{n}}$ denote feature space mappings corresponding to the kernel function $\kappa_{n}(\mathbf{a}, \mathbf{b}):=<\phi_{n}(\mathbf{a}), \phi_{n}(\mathbf{b})>$. We use a compact representation of the fMRI signal $\mathbf{x}_{i}$ in feature spaces $\Phi_{n_{i}}:=\left[\phi_{n_{i}}\left(\mathbf{x}_{i 1}\right), \ldots, \phi_{n_{i}}\left(\mathbf{x}_{i T}\right)\right]^{\prime}$ is of dimension $T \times n_{i}$. Denote by $\mathbf{K}_{i}=\Phi_{n_{i}} \Phi_{n_{i}}^{\prime}$ a $T \times T$ inner product kernel matrix. We consider the following kernel canonical correlation estimates:

$\hat{\rho}_{c}(i, j)=\max _{\boldsymbol{w}_{i} \in \mathbb{R}^{T}, \boldsymbol{w}_{j} \in \mathbb{R}^{T}} \boldsymbol{w}_{i}^{\prime} \mathbf{K}_{i}^{\prime} \mathbf{K}_{j} \boldsymbol{w}_{j} \quad$ subject to $\quad \boldsymbol{w}_{i}^{\prime} \mathbf{K}_{i}^{\prime} \mathbf{K}_{i} \boldsymbol{w}_{i} \leq 1, \quad \boldsymbol{w}_{j}^{\prime} \mathbf{K}_{j}^{\prime} \mathbf{K}_{j} \boldsymbol{w}_{j} \leq 1$,

This is equivalent to solve the following equation:

$$
\left\{\begin{array}{l}
\left(\mathbf{K}_{j}^{\prime} \mathbf{K}_{j}\right)^{-1} \mathbf{K}_{j}^{\prime} \mathbf{K}_{i}\left(\mathbf{K}_{i}^{\prime} \mathbf{K}_{i}\right)^{-1} \mathbf{K}_{i}^{\prime} \mathbf{K}_{j} \boldsymbol{w}_{j}=\lambda^{2} \boldsymbol{w}_{j} \\
\left(\mathbf{K}_{i}^{\prime} \mathbf{K}_{i}\right)^{-1} \mathbf{K}_{i}^{\prime} \mathbf{K}_{j}\left(\mathbf{K}_{j}^{\prime} \mathbf{K}_{j}\right)^{-1} \mathbf{K}_{j}^{\prime} \mathbf{K}_{i} \boldsymbol{w}_{i}=\lambda^{2} \boldsymbol{w}_{i}
\end{array}\right.
$$

$\mathbf{K}_{i}$ and $\mathbf{K}_{j}$ are not necessarily nonsingular, so it has been suggested to add a regularization term in the equation (Nielsen et al. 1998, Leurgans et al. 1993, Fukumizu et al. 2007), i.e.

$$
\left\{\begin{array}{l}
\left(\mathbf{K}_{j}^{\prime} \mathbf{K}_{j}+\gamma \mathbf{I}\right)^{-1} \mathbf{K}_{j}^{\prime} \mathbf{K}_{i}\left(\mathbf{K}_{i}^{\prime} \mathbf{K}_{i}+\gamma \mathbf{I}\right)^{-1} \mathbf{K}_{i}^{\prime} \mathbf{K}_{j} \boldsymbol{w}_{j}=\lambda^{2} \boldsymbol{w}_{j} \\
\left(\mathbf{K}_{i}^{\prime} \mathbf{K}_{i}+\gamma \mathbf{I}\right)^{-1} \mathbf{K}_{i}^{\prime} \mathbf{K}_{j}\left(\mathbf{K}_{j}^{\prime} \mathbf{K}_{j}+\gamma \mathbf{I}\right)^{-1} \mathbf{K}_{j}^{\prime} \mathbf{K}_{i} \boldsymbol{w}_{i}=\lambda^{2} \boldsymbol{w}_{i}
\end{array}\right.
$$


where $\gamma$ is the regularization parameter. This is equivalent to maximizing

$$
\frac{\boldsymbol{w}_{i}^{\prime} \mathbf{K}_{i}^{\prime} \mathbf{K}_{j} \boldsymbol{w}_{j}}{\sqrt{\boldsymbol{w}_{i}^{\prime} \mathbf{K}_{i}^{\prime} \mathbf{K}_{i} \boldsymbol{w}_{i}+\gamma \boldsymbol{w}_{i}^{\prime} \boldsymbol{w}_{i}} \sqrt{\boldsymbol{w}_{j}^{\prime} \mathbf{K}_{j}^{\prime} \mathbf{K}_{j} \boldsymbol{w}_{j}+\gamma \boldsymbol{w}_{j}^{\prime} \boldsymbol{w}_{j}}}
$$

The parameter $\gamma$ controls over-fitting and enhances the numerical stability. The correlation $\rho_{c}(i, j)$ yields a measure of brain connectivity between regions $i$ and $j$, taking into account the variation among all the voxels in each region. This is more informative than the simple correlation estimates between region-level summary statistics. The weights $\boldsymbol{w}_{i}$ can be used to determine the influence of various voxels on the regional correlation measure. The details on the procedure of solving (6) to obtain the kernel canonical correlation coefficient can be found in Fukumizu et al. (2007) and Kuss and Graepel (2003).

\subsection{A permutation test to construct the brain network}

To construct brain networks, we utilize a permutation test for the hypothesis testing problem: $H_{0}: \rho_{c}(i, j)=0$, versus $H_{1}: \rho_{c}(i, j) \neq 0$, for each region pair $(i, j)$ for $1 \leq i<j \leq p$. We permute the data over $\{1,2, \ldots, T\}$ scans, yielding a total of $L$ permutations, indexed by $l=1,2, \ldots, L$. Let $\mathbf{X}_{i}^{(l)}$ be the permuted data set and $\mathbf{K}_{i}^{(l)}$ be the corresponding inner product kernel matrix. The permuted kernel canonical correlation is estimated by

$$
\widehat{\rho}_{c}^{(l)}(i, j)=\max _{\boldsymbol{w}_{i} \in \mathbb{R}^{T}, \boldsymbol{w}_{j} \in \mathbb{R}^{T}} \boldsymbol{w}_{i}^{\prime} \mathbf{K}_{i}^{(l)^{\prime}} \mathbf{K}_{j} \boldsymbol{w}_{j} \quad \text { subject to } \quad \boldsymbol{w}_{i}^{\prime} \mathbf{K}_{i}^{(l)^{\prime}} \mathbf{K}_{i} \boldsymbol{w}_{i} \leq 1, \boldsymbol{w}_{j}^{\prime} \mathbf{K}_{j}^{\prime} \mathbf{K}_{j} \boldsymbol{w}_{j} \leq 1
$$

The permuted $p$ value is obtained by

$$
\widehat{p}(i, j)=\frac{1}{L} \sum_{l=1}^{L} I\left[\left|\widehat{\rho}_{c}^{(l)}(i, j)\right|>\left|\widehat{\rho}_{c}(i, j)\right|\right],
$$

where $I[\mathcal{A}]=1$ if event $\mathcal{A}$ occurs and $I[\mathcal{A}]=0$ otherwise. Each $\widehat{p}(i, j)$ is corrected by controlling of the false discovery rate in multiple testing under dependency (Benjamini and Yekutieli 2001), denoted $\widetilde{p}(i, j)$. The edge $(i, j)$ is included in the brain network indicating a strong functional connection between regions $i$ and $j$, if $\widetilde{p}(i, j)<\alpha$. 


\subsection{Brain connectivity analysis for multiple subjects}

Many neuroimaging studies seek to determine a group level brain network; and perhaps compare the networks between two or more groups of subjects, e.g. the MDD network and the $\mathrm{HC}$ network. In this work, we propose a very simple voting strategy to produce the group level brain network inference. To be more specific, using the permutation approach in Section 2.2, we can obtain the subject-specific network. Denote by $e_{s}(i, j) \in\{0,1\}$ the edge $(i, j)$ inclusion indicator for subject $s=1, \ldots, S$, then the group level edge inclusion indicator for the whole group is estimated by

$$
E_{\zeta}(i, j)=I\left[\frac{1}{S} \sum_{s=1}^{S} e_{s}(i, j)>\zeta\right] \text {, for } \zeta \in(0,1)
$$

Thus, a connection in the group-level network is deemed to be present if the proportion of subject-level connections exceeds a specified threshold $\zeta$. Compared with other strategies, the proposed voting strategy has two main advantages: 1) it requires small computation costs for group level analysis, and it is straightforward to

implement parallel computing; 2) It is robust to outliers and can accommodate the noisy and heterogeneous fMRI data. Based on the empirical results that we show in the simulation studies and our application to the depression data in the following sections, this procedure exibits good performance.

\section{Characterizing a Depression-Related Brain Net- work}

We apply our method to resting-state fMRI data from 20 MDD patients and 20 healthy control (HC) subjects in the Predictors of Remission in Depression to Individual and Combined Treatments (PReDICT) study (Dunlop et al. 2012). The HC subjects and the MDD patients are matched by age and gender. The average age is 45.8 years for the MDD group, and 42.4 years for the controls; both groups are 
$50 \%$ male. In the MDD group, the average Hamilton Depression Rating Scale Score (HAM-D) is 19 and average length of the major depressive episode is 82 weeks. All the MDD patients have unipolar depression and have never been treated. For each subject, 150 fMRI volumes are acquired using a three-Tesla whole-body MR system for 7.5 minutes (Siemens MAGNETOM TIM Trio, Siemens Medical Solutions, Erlangen, Germany) (Dunlop et al. 2012).

By using the preprocessing script based on the Configurable Pipeline for the Analysis of Connectomes (C-PAC), we performed standard preprocessing steps including: motion correction, slice timing correction, normalization and spatial smoothing. Also, we applied de-trending and demeaning steps to remove the incoherent background shift and texture variation. All the images were registered in $2 \mathrm{~mm}$ MNI space with size $91 \times 109 \times 91$. Our analysis focused on constructing the whole brain region level network which consists of 90 AAL regions with 185,405 voxels. The full names and short names of the AAL regions are listed in the Appendix.

\subsection{Consistent functional connections and regions}

Applying methods in Section 2, we construct the brain network for each of the 20 MDD patients and $20 \mathrm{HC}$ subjects. For each subject, the regularization parameter $\gamma$ is chosen as 0.1 based on the default settings from the R package kernlab (Kuss and Graepel 2003) . Histograms of connections for different groups are shown in Figure 1. Overall, the MDD patients have more functional connections compared to the HC subjects. We apply (8) to construct the group level brain functional networks for MDD patients and $\mathrm{HC}$ subjects respectively. We vary the parameter $\zeta$, which reflects the proportion of subjects who have consistent connections, from 0.5 to 1.0 , to assess the heterogeneity of the number of connections in the subject level networks within each group. The number of consistent connections and consistent regions are summarized in Figure 2, showing that the MDD patients have a slightly higher 
number of consistent connections and regions compared to the $\mathrm{HC}$ patients; and the number of consistent connections and regions both decrease linearly as $\zeta$ increases.

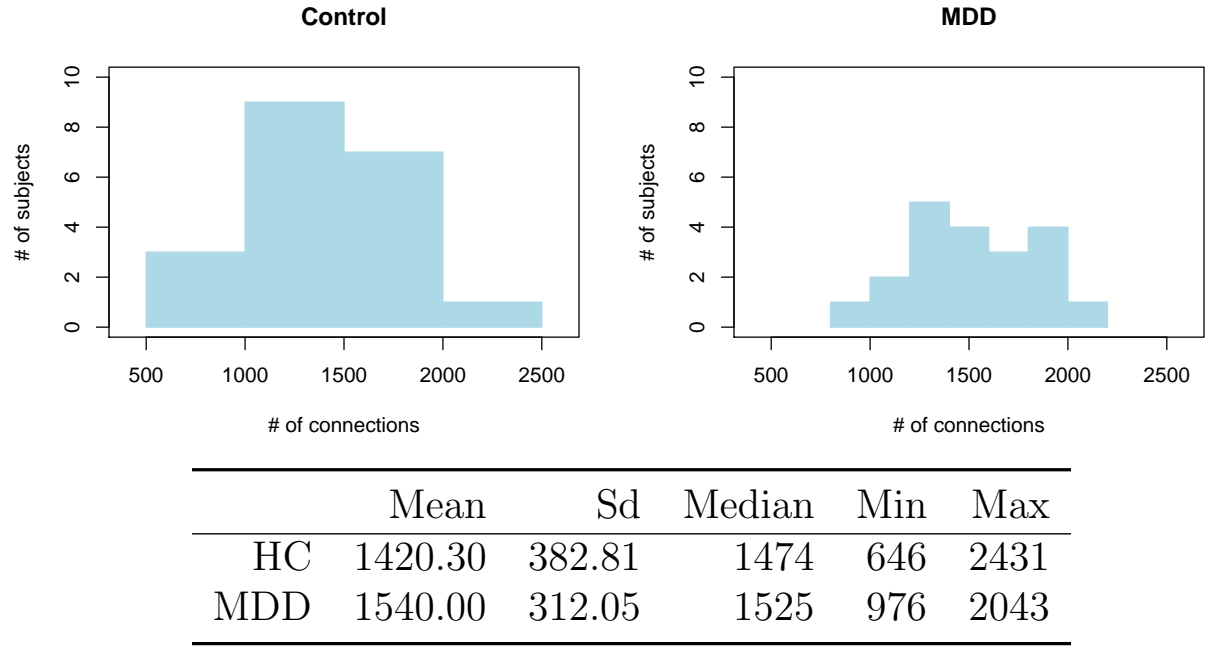

Figure 1: Histogram and summary statistics of the number of functional connections in the MDD patient network and the HC subject network.

The numbers of perfectly consistent connections (i.e. $\zeta=1$, where all the subjects within the group have the connection) are 33 and 25 for MDD patients and HC subjects, respectively. Among those, 17 connections (involving 19 ROIs) exist in all 40 subjects across the two groups, based on which we define a common network. There are 8 connections that appear in the $\mathrm{HC}$ subjects but not for all MDD patients, which defines the distinct $\mathrm{HC}$ network. The ROIs in the common network and the HC network involve several core regions in the brain's default mode network (Buckner et al. 2008). Also, the MDD patients have 16 connections that do not appear consistently for all the $\mathrm{HC}$ subjects, to which we refer as the distinct MDD network. These three networks are summarized in Figure 3. Table 1 summarizes proportions of $\mathrm{HC}$ subjects and MDD patients exhibiting the functional connections for each region pair in the MDD network and the $\mathrm{HC}$ network, respectively. A binomial test is used to determine the difference in proportions between the two groups. For example, all 20 (100\%) MDD patients develop function connections between the left 
supramarginal gyrus (SMG.L) and the right median cingulate and paracingulate gyri (DCG.R), where only 13 (65\%) HC subjects have this connection. The binomial test indicates that this difference is significant (the adjusted p-value $=0.013$ ) with the control of false discovery rate..

Table 1: Proportions of HC subjects and MDD patients exhibiting the functional connections for each region pair in the MDD network and the HC network, respectively. Binomial tests are performed to determine the difference in proportion between MDD patients and HC subjects for each region pair.

\begin{tabular}{|c|c|c|c|c|c|}
\hline \multicolumn{3}{|c|}{$\mathrm{MDD}=1.00$} & \multicolumn{3}{|c|}{$\mathrm{HC}=1.00$} \\
\hline Region Pair & $\mathrm{HC}$ & $\mathrm{P}_{\text {-value }}^{\dagger}$ & Region Pair & MDD & P-value ${ }^{\dagger}$ \\
\hline PCG.L-ORBsup.L & 0.90 & 1.000 & PoCG.L-PreCG.R & 0.85 & 1.000 \\
\hline SMG.L-ORBsup.L & 0.90 & 1.000 & PCG.R-ORBsup.L & 0.95 & 1.000 \\
\hline PAL.L-ORBsup.L & 0.85 & 0.547 & CUN.R-ORBsup.R & 0.95 & 1.000 \\
\hline AMYG.L-ORBsup.R & 0.90 & 1.000 & STG.L-ORBmid.L & 0.95 & 1.000 \\
\hline CAL.R-ORBsup.R & 0.80 & 0.387 & MOG.L-ORBmid.R & 0.95 & 1.000 \\
\hline OLF.L-MFG.R & 0.95 & 1.000 & PAL.R-REC.R & 0.95 & 1.000 \\
\hline PoCG.L-MFG.R & 0.95 & 1.000 & THA.L-INS.L & 0.95 & 1.000 \\
\hline ANG.L-MFG.R & 0.85 & 0.547 & PAL.L-ACG.L & 0.95 & 1.000 \\
\hline STG.R-ORBmid.L & 0.80 & 0.387 & & & \\
\hline ORBsup.R-REC.R & 0.90 & 1.000 & & & \\
\hline MFG.L-REC.R & 0.95 & 1.000 & & & \\
\hline OLF.R-REC.R & 0.90 & 1.000 & & & \\
\hline PreCG.R-INS.L & 0.85 & 0.547 & & & \\
\hline PoCG.R-ACG.R & 0.95 & 1.000 & & & \\
\hline SPG.R-ACG.R & 0.95 & 1.000 & & & \\
\hline SMG.L-DCG.R & 0.65 & 0.013 & & & \\
\hline
\end{tabular}

${ }^{\dagger} \mathrm{P}$-values have been adjusted by the control of the false discovery rate in multiple testing under dependency (Benjamini and Yekutieli 2001)

\subsection{Difference between the HC and the MDD network}

As shown in the previous section, based on the permutation testing results, we obtain the multi-attribute network for each of the $\mathrm{HC}$ subjects and the MDD patients, denoted $e_{s}(i, j)$, for $s=1, \ldots, S$ and $1 \leq i<j \leq 90$. We introduce $d_{s}$ to represent the disease status for each subject $s$, where $d_{s}=0$ if the subject is a HC and $d_{s}=1$ if the subject is an MDD. Considering $\left\{d_{s}, e_{s}(i, j)\right\}$ as the observed data, we are 

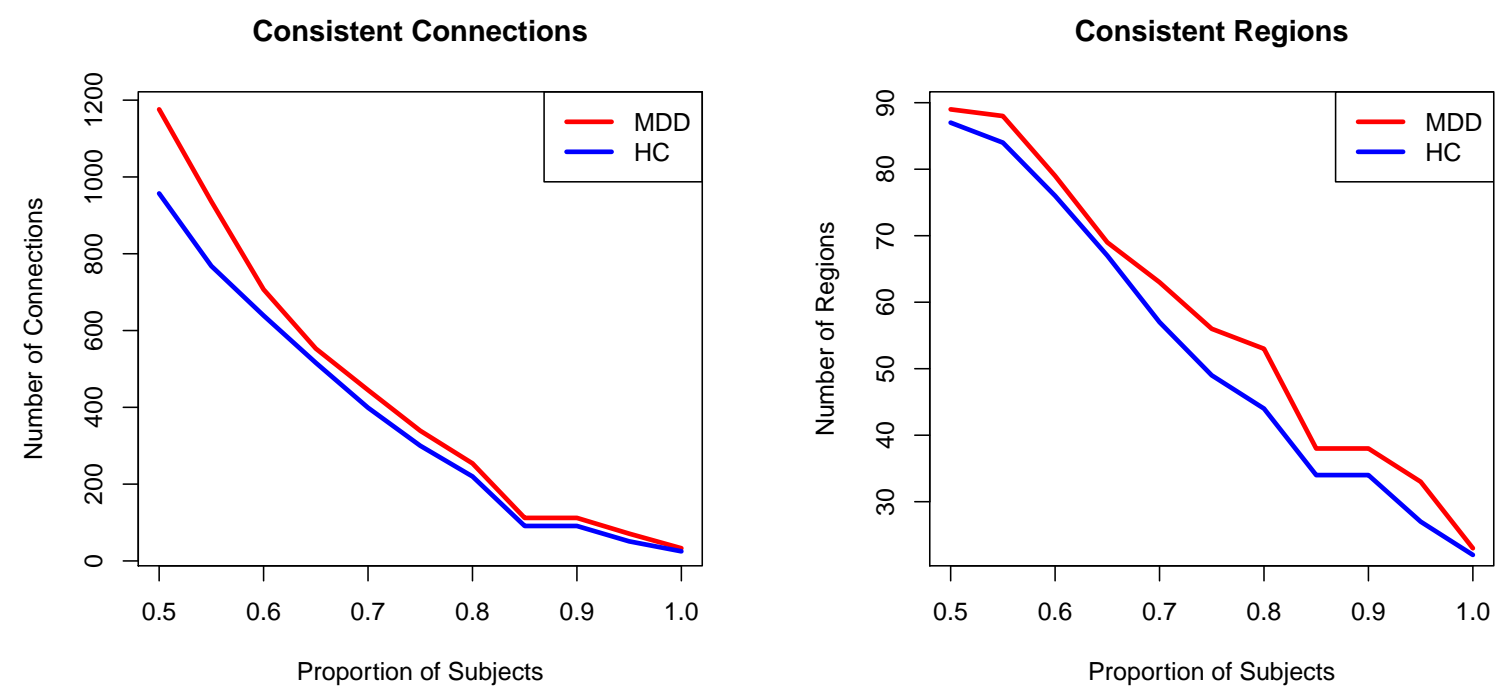

Figure 2: Number of consistent connections and regions across brain functional networks of subjects in MDD and HC groups
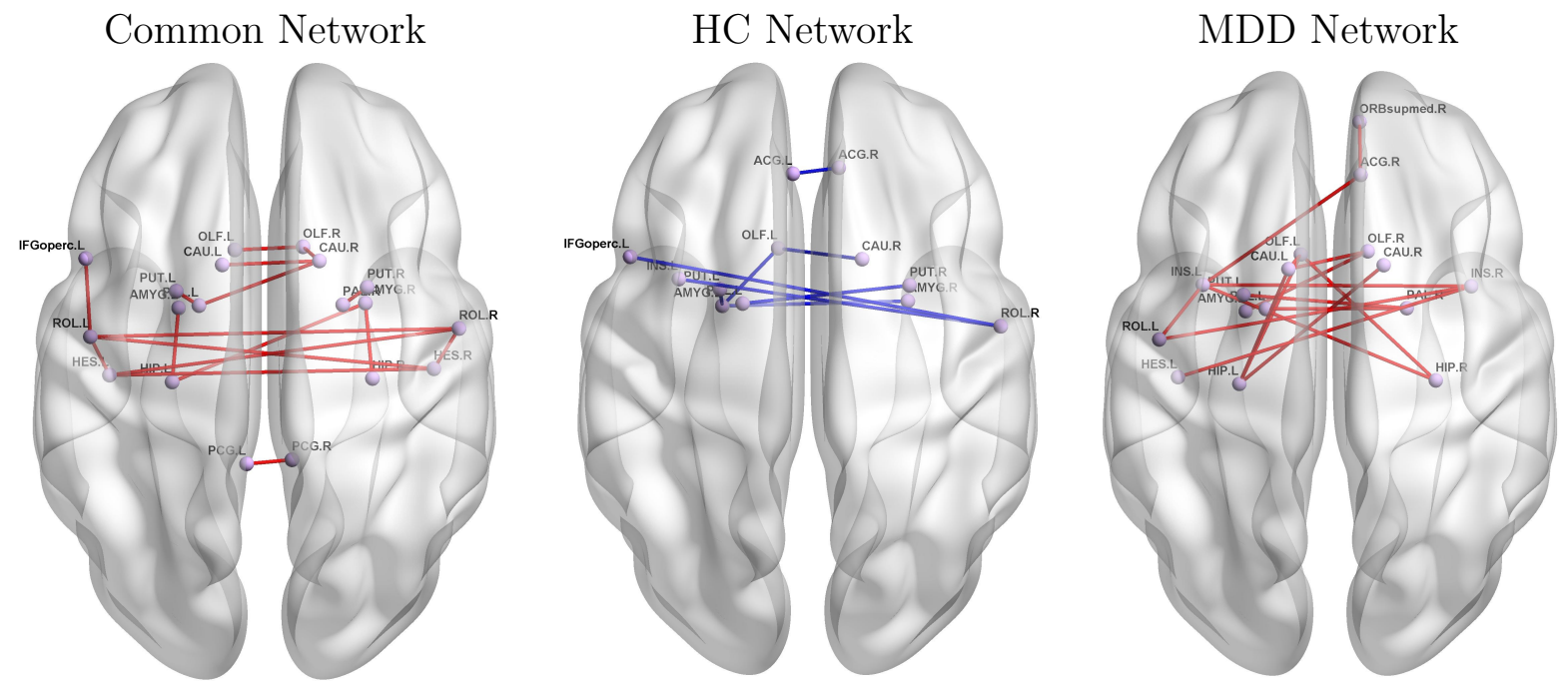

Figure 3: Functional networks with consistent connections for all subjects within each group $(\zeta=1)$. The common network consists of connections that are consistent across all subjects in both groups. The HC network is consistent for all control subjects, but not for MDD subjects. The MDD network shows consistent connections for MDD patients, which were not consistently observed across all HC subjects. 
interested in detecting difference in functional network between the two groups. For $s=1, \ldots, 20$, we can approximately have

$$
\left[e_{s}(i, j) \mid d_{s}=g\right] \sim \operatorname{Bernouli}\left[p_{g}(i, j)\right]
$$

for each $g=0,1$ and $1 \leq i<j \leq 90$, where $p_{g}(i, j)$ represents the probability of regions $i$ and $j$ being connected for group $g$. To construct the difference network, we consider the following multiple hypothesis testing problem: for all $(i, j)$ with $1 \leq i<i \leq 90$

$$
H_{0}^{(i, j)}: p_{0}(i, j)=p_{1}(i, j) \quad \text { versus } \quad H_{1}^{(i, j)}: p_{0}(i, j) \neq p_{1}(i, j)
$$

where the null hypothesis $H_{0}^{(i, j)}$ implies that there is no group difference in the functional connectivity between regions $i$ and $j$. We perform a total of 4,005 (number of region pairs out of 90 regions) binomial tests. We have adjusted the p-values by the control of the false discovery rate in multiple testing under dependency (Benjamini and Yekutieli 2001). In particular, we used the $\mathrm{R}$ function p.adjust with option method=BY. and the significance level was set to 0.01 . We identify 35 region pairs showing significant differences in functional connectivity between the $\mathrm{HC}$ subjects and MDD patients. Among those, there are only two region pairs: IFGoperc.LROL.R and ANG.L-ROL.R where the proportions of MDD patients having the corresponding functional connections are much smaller than those of the HC subjects, indicating the reduced functional connectivity, while for the rest of regions pairs, the MDD patients show increased functional connectivity relative to the $\mathrm{HC}$ subjects. The table in Figure 4 summarizes the eleven region pairs with the largest differences between the two groups in the proportion of subjects exhibiting strong functional connections. In particular, a large proportion of $\mathrm{HC}$ subjects $(85 \%)$ exhibit functional connection between the region pair ROL.R-ANG.L. In contrast, a large proportion of MDD patients (70\%) have strong functional connectivities between the region pair 
CAL.R-THA.R. The remaining region pairs in the table where approximately half MDD patients and $5 \%$ or fewer of the $\mathrm{HC}$ subjects demonstrate this pattern of the functional connectivity. The presence of some less significant patterns that still discriminate the MDD pts from the HC may suggest further MDD subgroups. The current dataset is insufficient to explore this possibility.

To identify important "hub" regions that have substantial differences between the two groups, we conduct an analysis of the network degree (i.e. the number of connections each node has to other nodes ) between the HC subjects and the MDD patients. For each node (ROI), we compute the degree in each of 40 subject specific networks. We provide a list of top five regions that exhibit largest group differences in degree along with associated summary statistics (mean and standard deviation) of the degree in the two groups (see Table 2).

Table 2: Regions with substantial difference in network degree between the HC subjects and the MDD patients

\begin{tabular}{crrrr}
\hline \multirow{2}{*}{ Region } & \multicolumn{2}{c}{ HC } & \multicolumn{2}{c}{ MDD } \\
\cline { 2 - 5 } & Mean & Sd & Mean & Sd \\
\hline Middle frontal gyrus, orbital part (R) & 33.10 & 13.10 & 41.90 & 14.00 \\
Parahippocampal gyrus (L) & 24.80 & 11.70 & 32.90 & 11.10 \\
Lingual gyrus (L) & 26.10 & 13.30 & 35.00 & 13.10 \\
Temporal pole: middle temporal gyrus (L) & 22.10 & 13.60 & 35.80 & 18.40 \\
Temporal pole: middle temporal gyrus (R) & 23.60 & 11.00 & 33.10 & 15.10 \\
\hline
\end{tabular}

\subsection{Network based prediction of depression risk}

To evaluate the performance of our proposed method on the identification of the functional network, we perform a classification analysis of the disease status using a network based support vector machine (NET-SVM) model. Denote by $\mathcal{D}$ the set of edges with significant difference in functional connectivity between the two groups. For example, in the previous section, we use the binomial test to identity 35 significant 


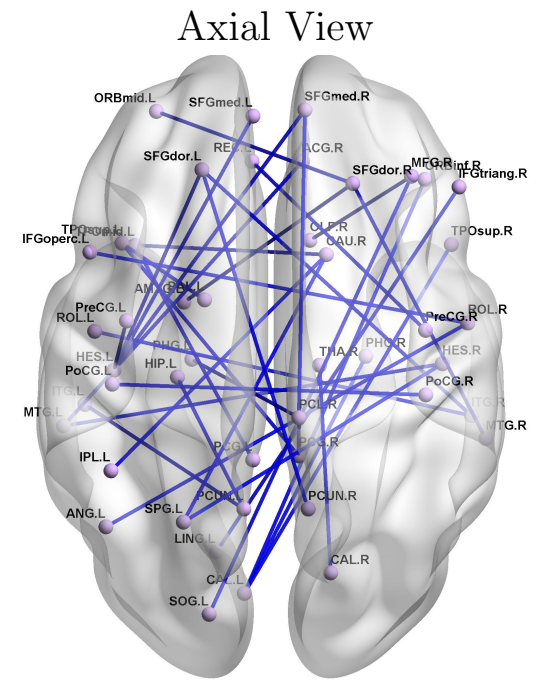

Left Sagittal View

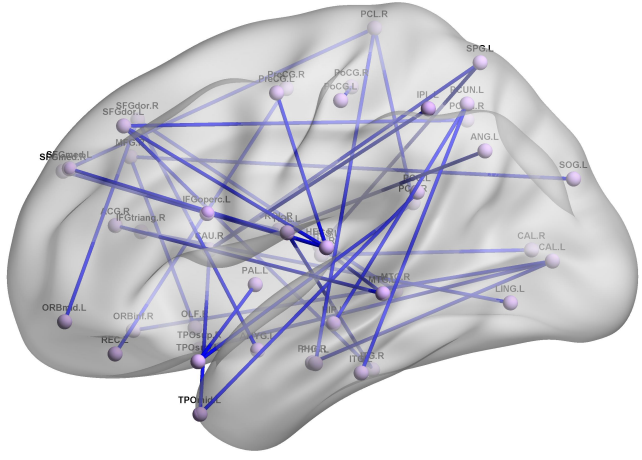

Coronal View

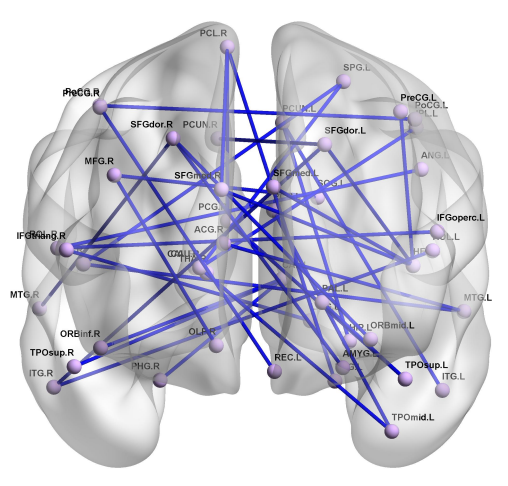

Right Sagittal View

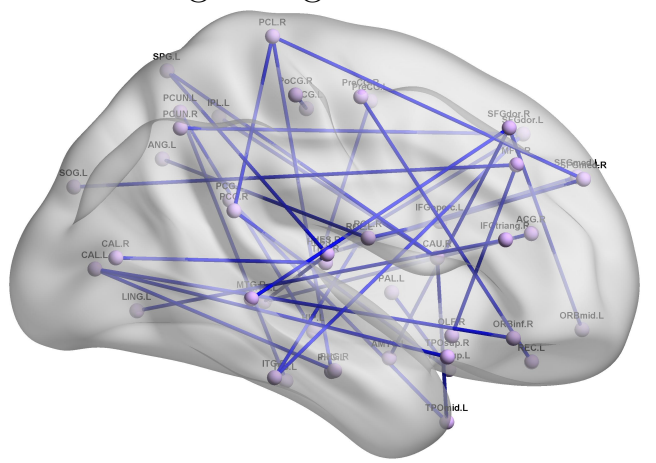

\begin{tabular}{cll}
\hline Region Pairs & HC & MDD \\
\hline ROL.R-ANG.L & $\mathbf{0 . 8 5}$ & $\mathbf{0 . 3 0}$ \\
IFGtriang.R-LING.L & 0.05 & 0.50 \\
MFG.R-SOG.L & 0.00 & 0.45 \\
SFGmed.R-PCL.R & 0.10 & 0.55 \\
PCG.R-PCL.R & 0.10 & 0.55 \\
PHG.L-PCL.R & 0.05 & 0.55 \\
CAL.R-THA.R & $\mathbf{0 . 2 0}$ & $\mathbf{0 . 7 0}$ \\
ACG.R-MTG.L & 0.10 & 0.55 \\
PCG.L-TPOmid.L & 0.10 & 0.55 \\
CAU.R-TPOmid.L & 0.05 & 0.50 \\
SFGdor.L-ITG.R & 0.05 & 0.50 \\
\hline
\end{tabular}

Figure 4: Network region pairs (edges) showing statistically significant differences in functional connectivity between the HC subjects and the MDD patients. The table summarizes the 11 region pairs with the largest differences between the two groups in the proportion of subjects exhibiting strong functional connections. All the corresponding adjusted p-values are less than 0.0001 . 
region paris. In the NET-SVM model, for each subject $s$, the predictor is a subset of edges on the multi attribute network, which is defined as $\mathbf{x}_{s}=\left\{e_{s}(i, j)\right.$, edge $\left.(i, j) \in \mathcal{D}\right\}$ and the outcome is the disease status $d_{s}$, for $s=1, \ldots, S$. We apply the standard least squares support vector machine classifiers (Suykens and Vandewalle 1999) to the data set $\left\{d_{s}, \mathbf{x}_{s}\right\}_{s=1}^{S}$. We choose the radial kernel $\exp \left(-|u-v|^{2}\right)$. We examine the prediction accuracy via leave-one-out cross validation (LOOCV), ten-fold CV and five-fold CV respectively. In particular, for ten fold and five fold cross validation, we randomly partition the 40 subjects into 36 plus 4 and 32 plus 8 , respectively, as the training and testing data sets. We repeat the above partitions over 1,000 times.

The true positives (TP) or the number of subjects that are correctly classified as MDD patients, false positives (FP) or the number of subjects that are incorrectly classified as MDD patients, true negatives (TN) or the number of subjects that are incorrectly classified as healthy controls, false negatives (FN) or the number of subjects that are incorrectly classified as healthy controls, sensitivity (SENS) or the proportion of the MDD patients being correctly identified, specificity (SPEC) or the proportion of healthy subjects being correctly identified, positive predictive value (PPV) or the ratio of true positives to combined true and false positives and negative predictive value (NPV) or the ratio of true negatives to combined true and false negatives are summarized in Table 3. For the ten-fold and the five fold results, the numbers are the average over 1,000 random partitions. The classification accuracy is consistently high for the different cross validation methods, where the LOOCV has the perfect classification accuracy, the five fold CV and the ten fold CV only show a slightly lower power and a very small false discovery rate compared with the LOOCV results. These results imply that the identified 35 kernel CCA functional connectivity biomarkers can almost perfectly classify the the depression disease status for the 40 subjects in our datasets. Those biomarkers can be considered as potentially important biomarkers 
for the future studies with larger sample size.

To demonstrate the benefit of the kernel CCA approach, we also apply the PCA measure to analyze the PReDICT data and keep all other procedures the same as the kernel CCA methods in terms of the multiple comparison procedure and the thresholding criteria. The PCA only identifies three important function connections. They are MFG.R - SOG.L, SFGmed.R - PCL.R and PCG.R - PCL.R, which are all identified by our kernel CCA measure (see Figure 4). Only using these three connections as the the predictors, we also apply the NET-SVM method and obtain a five fold cross validation prediction accuracy of 0.82 (based on 1,000 random splitting the dataset), much smaller than 0.99, the prediction accuracy provided by the kernel CCA. This indicates that the functional connectivity biomarkers that are defined by the PCA measure are not as good as those based on the kernel CCA measure in terms of separating the depression patients from the healthy controls in our dataset.

Table 3: Cross validation classification accuracy of the NET-SVM model

\begin{tabular}{rrrrrrrrr}
\hline & TP & TN & FP & FN & SENS & SPEC & PPV & NPV \\
\hline LOOCV & 20 & 20 & 0 & 0 & 1.000 & 1.000 & 1.000 & 1.000 \\
Ten-Fold CV & 1.997 & 2.001 & 0.002 & 0.000 & 1.000 & 0.999 & 0.999 & 1.000 \\
Five-fold CV & 4.046 & 3.937 & 0.017 & 0.000 & 1.000 & 0.996 & 0.996 & 1.000 \\
\hline
\end{tabular}

\section{Simulation Studies}

We conduct two simulation studies to evaluate the performance of the proposed method for detecting the functional connectivity between a region pair and constructing the functional network among multiple regions.

\subsection{Functional connectivity between two regions}

We start with a simple illustration of the proposed method to detect the functional connectivity between two regions. We simulate two types of fMRI signals: indepen- 
dent signals, i.e. the voxel-wise signals are mutually independent across all the voxel pairs between the two regions; and dependent signals: the voxel-wise signals are associated with each other across the two regions, where the corresponding associations are characterized by Gaussian processes (GPs). Specifically, the number of voxels are set to $n_{1}=500$ and $n_{2}=250$, respectively. The number of scans $T$ is set to 150 . For $i=1,2, t=1, \ldots, T$ and $k=1, \ldots, n_{i}$. We have

- Independent fMRI signals: draw $x_{i t k} \stackrel{\text { iid }}{\sim} \mathrm{N}(0,1)$, where "iid" represents random variables are independent and identically distributed.

- Dependent fMRI signals: let $x_{i t k}=\sum_{l=1}^{100} g_{i k l}\left(z_{t l}\right)$, where $z_{t l} \stackrel{\text { iid }}{\sim} \mathrm{U}[0,1]$ and $g_{i k l} \stackrel{\text { iid }}{\sim}$ $\mathcal{G P}\left(z_{1 l}, \kappa\right)$. $\mathcal{G P}(\mu, \kappa)$ denotes a Gaussian process with mean $\mu$ and covariance kernel $\kappa$ on time interval $[0,1]$. We take $\kappa\left(s, s^{\prime}\right)=\exp \left\{-10\left(s-s^{\prime}\right)^{2}\right\}$. The GP $g_{i k l}$ can model temporal correlations over scans. Specifically, for any two time points $t$ and $t^{\prime}$, we have $\operatorname{cov}\left(x_{i t k}, x_{i t^{\prime} k}\right)=100 \int_{0}^{1} \int_{0}^{1} \exp \left[-10\left(s-s^{\prime}\right)\right] \mathrm{d} s \mathrm{~d} s^{\prime}$ and $\operatorname{cov}\left(x_{1 t k}, x_{2 t k}\right)=100 \operatorname{var}\left(z_{11}\right)=25 / 3$.

We make comparisons between two metrics: the correlation between the first principal component scores (PCA) and the first kernel canonical correlation coefficient (kCCA). Figure 5 shows the permutation distribution of three test statistics and the corresponding p-values for the two cases in one simulated dataset. The results imply that the kCCA produces small p-value $(<0.01)$ for the dependent case, correctly identifying the non-linear association between the two regions, while they both generate large p-values for the independent cases.

We repeatedly generate the 200 simulated datasets for each of the independent and dependent cases. We summarize the permutation testing performance at significance of 0.05. We compute the power, the type I error and the false discovery rate (FDR) in Table 4. The results show that the proposed kernel CCA metric provided optimal power of detecting the functional connectivity between the two regions, and it well 
$\mathrm{kCCA}$
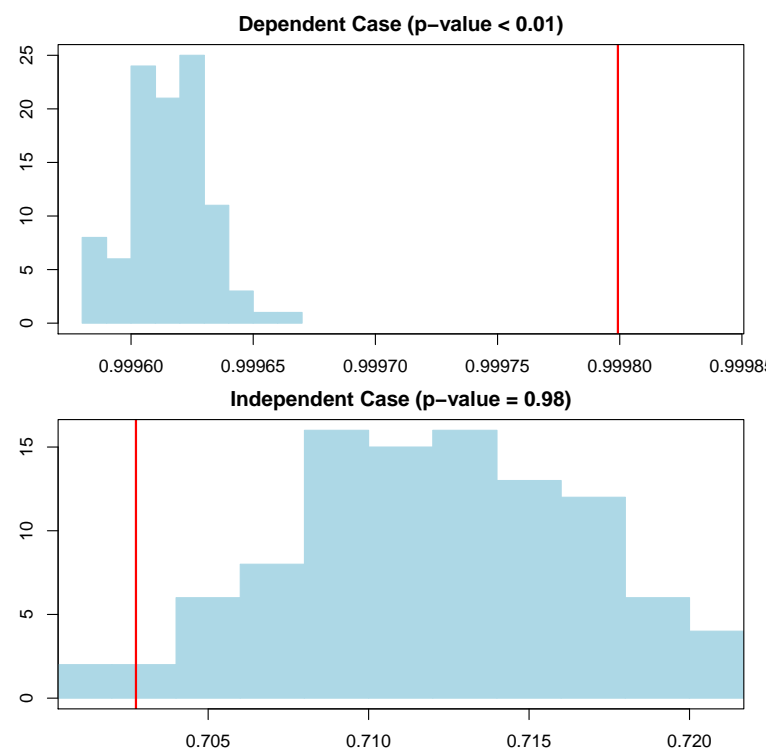

PCA
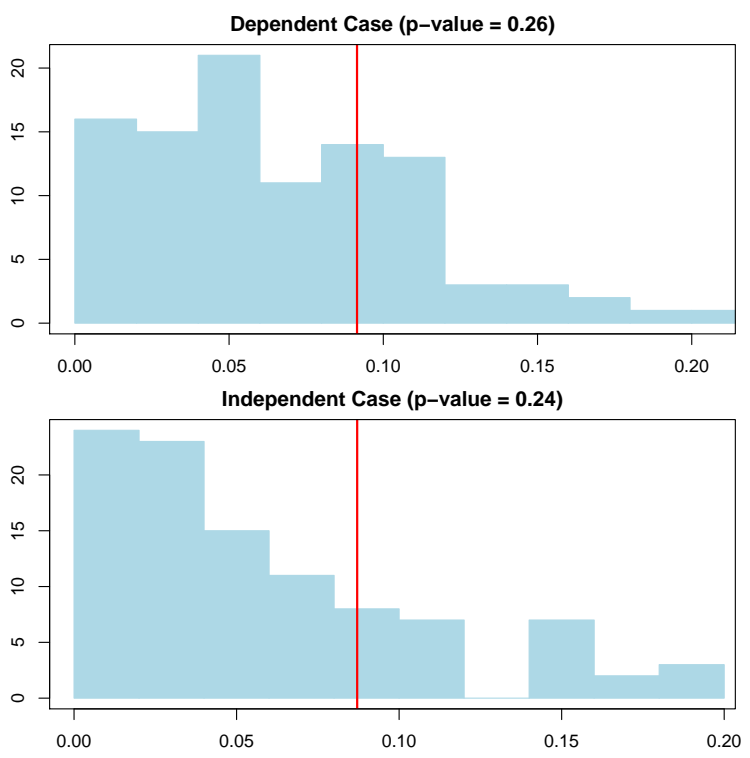

Figure 5: Simulation studies that compare the permutation distributions of the two region-level connectivity metrics (kCCA and PCA) for both dependent and the independent cases.

controls the type I error and the false discovery rate (FDR); while the PCA approach has lower power and an inflated FDR.

Table 4: Permutation test performance via different metrics over 200 simulated datasets: kernel CCA versus sparse PCA (TP: True Positive, FP: False Positive, FN: False Negative, TN: True Negative)

\begin{tabular}{rrrrrrrr}
\hline & TP & FP & FN & TN & Power & Type I Error & FDR \\
\hline kCCA & 200 & 10 & 0 & 190 & 1.00 & 0.05 & 0.05 \\
sPCA & 40 & 8 & 160 & 192 & 0.20 & 0.04 & 0.17 \\
\hline
\end{tabular}

\subsection{Functional connectivity network over multiple regions}

We evaluate the accuracy of constructing functional brain networks over multiple regions of interests (ROIs) using the proposed method. In particular, we simulate two types of graphs on 30 ROIs: random graphs with edge probability 0.2 and scale free graphs, respectively, based on the Erdos-Renyi model and the Barabasi-Albert model. The simulated region-level networks are shown in Figure 6. We assume that 
the number of voxels per region is generated by a Poisson distribution with a mean of 200, i.e. $n_{i} \sim \operatorname{Poisson}(200)$, for $i=1, \ldots, 30$. The simulated $n_{i}$ range from 174 to 224 . Denote by $\boldsymbol{\Delta}=\left(\delta_{i j}\right)$ the adjacency matrix of the simulated network, where $\delta_{i j} \in\{0,1\}$ indicates whether regions $i$ and $j$ are functionally connected or not. Given a simulated $\boldsymbol{\Delta}$, the fMRI time series $x_{i t k}$ are generated by computing $y_{i t k}+\sum_{j=1}^{30} \delta_{i j} f_{j k}\left(z_{t}\right)$, where $y_{i t k} \sim \mathrm{N}(0,1), z_{t} \sim \mathrm{U}(0,1)$ and $f_{i k} \sim \mathcal{G P}(0, \kappa)$, where $\kappa\left(s, s^{\prime}\right)=\exp \left\{-10\left(s-s^{\prime}\right)^{2}\right\}$.

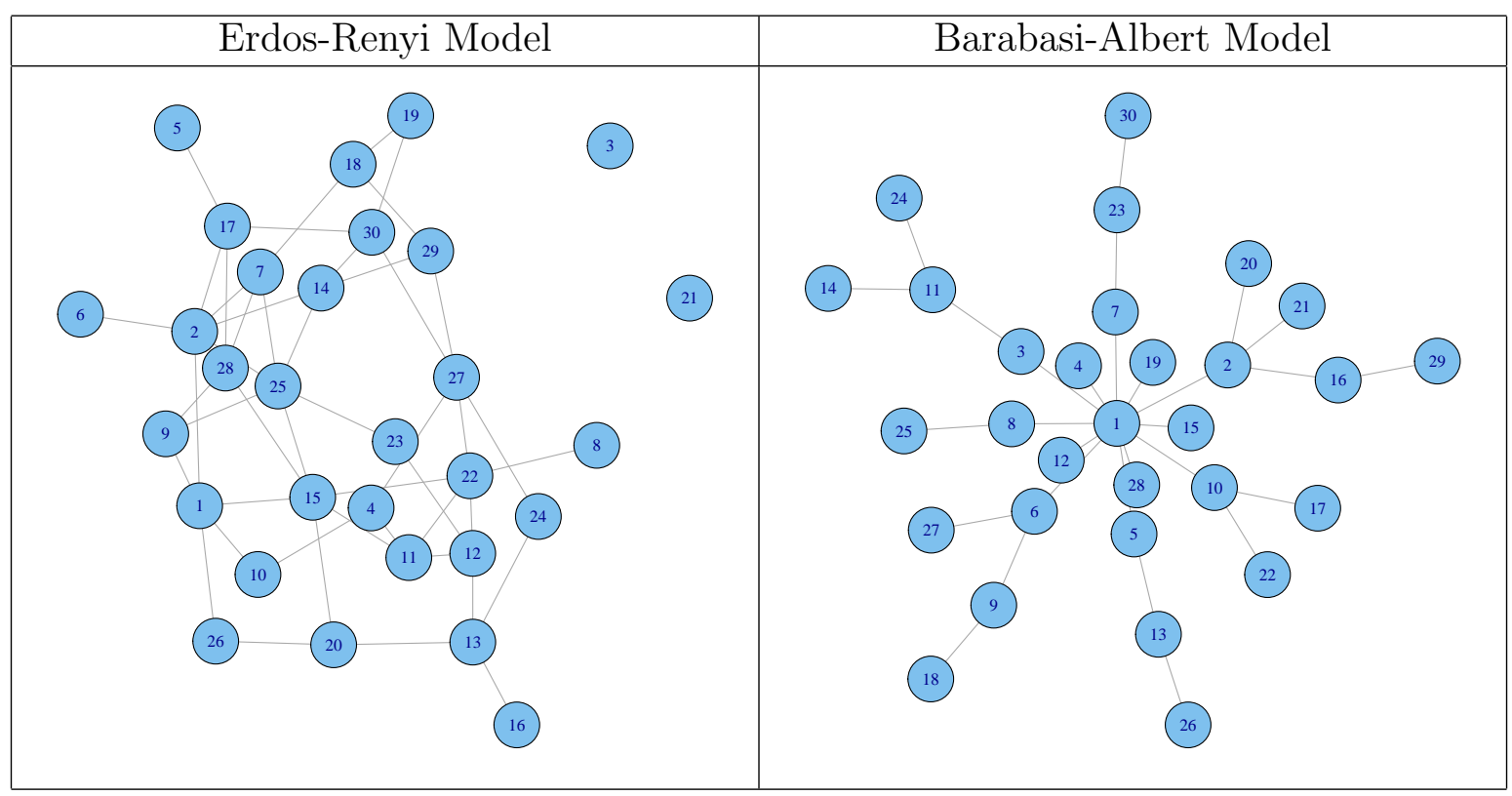

Figure 6: Two simulated networks: a random network (47 edges) based on the ErdosRenyi model and a scale free network (29 edges) based on the Barabasi-Albert model. The vertex represents the ROI; and an edge represents the two regions are functionally connected.

We generate a total of 200 simulated datasets for both network models and apply the proposed permutation test with $\mathrm{kCCA}$ and sPCA metrics to each dataset. We also consider a simple alternative approach by taking the average of the voxel-wise time series within each region and computing the correlation between region specific statistics. We refer to this approach as "meanCor". We summarize the accuracy 
of edge estimation in Table 5. The kCCA metric provides much higher power and better control of the FDR, compared to sPCA and meanCor which has low power and results in an inflated FDR. Also, we summarize the whole network estimation accuracy for the two metrics in Table 6 using the proportion of estimated networks that exactly recover the true simulated network, along with the proportion of the estimated networks that recover a certain percentage of the edges in the true network. We vary the percentages from $85 \%$ to $100 \%$. The results suggest that $28 \%$ of scale free networks and $21 \%$ random networks are fully recovered by the kCCA approach, while there are no sPCA estimated networks that are exactly identical to the corresponding true networks. If we allow up to $5 \%$ errors, i.e. at least $95 \%$ of true edges are included in the network, then over $90 \%$ of both scale free networks and random networks can be recovered by the kCCA approach, while the sPCA still cannot correctly identify any networks. Almost all the scale free networks and the random networks are recovered in the sense that at least $85 \%$ of true edges are included in the network. These results suggest that $\mathrm{kCCA}$ is much more powerful and accurate than the sPCA and meanCor in terms of estimating the network.

Table 5: Average of the edge estimation accuracy over 200 simulated datasets for the scale free network and the random network

\begin{tabular}{rrrrrrrr}
\multicolumn{8}{c}{ Scale free network } \\
\hline Method & TP & FP & FN & TN & Power & Type I error & FDR \\
\hline kCCA & 27.98 & 0.23 & 1.01 & 405.77 & 0.96 & 0.00 & 0.01 \\
sPCA & 17.82 & 4.29 & 11.38 & 401.52 & 0.61 & 0.01 & 0.19 \\
meanCor & 0.65 & 4.12 & 28.35 & 401.88 & 0.02 & 0.01 & 0.85
\end{tabular}

Random network with connection probability 0.2

\begin{tabular}{rrrrrrrr}
\hline Method & TP & FP & FN & TN & Power & Type I error & FDR \\
\hline kCCA & 45.83 & 0.39 & 1.18 & 387.62 & 0.97 & 0.00 & 0.01 \\
sPCA & 26.95 & 2.27 & 20.08 & 385.70 & 0.57 & 0.01 & 0.08 \\
meanCor & 1.02 & 3.96 & 45.98 & 384.05 & 0.02 & 0.01 & 0.80
\end{tabular}


Table 6: Network estimation accuracy over 200 simulated data sets: the frequency of the estimated networks including a certain percentage of edges in the true network (Pet), and the proportion of the estimated networks that exactly recover the true network (Exact). Note that a Pet of $100 \%$ does not imply that the estimated network exactly recover the true network, it may include a small number of false positive edges.

\begin{tabular}{lrrrrrr}
\hline & & \multirow{4}{c}{ Pet } \\
\cline { 4 - 7 } & & Exact & & \\
\cline { 4 - 7 } Scale free network & kCCA & 0.28 & 0.75 & 0.90 & 0.98 & 0.99 \\
& sPCA & 0.00 & 0.01 & 0.01 & 0.04 & 0.05 \\
& meanCor & 0.00 & 0.00 & 0.00 & 0.00 & 0.00 \\
Random network & kCCA & 0.21 & 0.30 & 0.91 & 0.98 & 1.00 \\
& sPCA & 0.00 & 0.00 & 0.00 & 0.01 & 0.01 \\
& meanCor & 0.00 & 0.00 & 0.00 & 0.00 & 0.00 \\
\hline
\end{tabular}

\section{Discussion}

We propose the use of multi-attribute canonical correlation graphs to perform functional brain network analysis at a region level, incorporating all the voxel level information. We perform simulation studies to demonstrate that our proposed method has advantages compared to existing methods using region level summary statistics in terms of network identification accuracy. We apply the proposed method to determine functional connectivity in a study of MDD. We identify important region pairs for which the $\mathrm{HC}$ subjects and the MDD patients have significantly different functional connectivity. Based on the difference network, we develop a network based classifier (NET-SVM) for making classification on the disease status. The cross validation results show that our method can achieve over $99 \%$ classification accuracy, which is much better than the existing alternative (a PCA based measure), indicating that the kernel CCA method is a better measure to classify the disease status in our study. Although a model that is an accurate classifier is not necessarily the ideal model to study pathophysiology, our model does provide a defensible, empirically based starting point for hypothesis driven follow up studies. In the future, our model can be 
used to answer other questions about depression such as further characterization of depression subtypes, response predictors, trait-state differences (sick versus well) and the like.

One limitation of our group analysis is that it is based on a relatively small number of subjects $(n=40)$. Methodologically, the small sample size is not an impediment to implementing our approach as it is applicable even at a single subject level. While we do not regard our group level results as definitive, based on the limited sample size, our simple voting strategy reveals that there are common functional connections. The identified group level functional connections for MDD patients are scientifically meaningful, some of which have been reported in the previous literature. We acknowledge the importance of reproducibility in imaging research generally, and our group-level results would accordingly benefit from additional inquiry.

Although the development of the proposed methods is motivated by the MDD study, it can be used to explore the functional connectivity networks for other neurological diseases, such as Parkinson's disease and Alzheimer's disease. The identified functional connections may provide evidence for researchers to determine the etiology of the associated disease. The proposed NET-SVM based classifier not only can be used for verifying the identified functional networks, but also it may provide an approach for investigating imaging guided diagnostics for associated disorders. Although our results reveal regional connections that show promise for accurately distinguishing MDD patients from $\mathrm{HC}$ subjects, that true generalizability of the predictive ability of these features will need to be borne out in applications to other data sets.

The current group level network estimation is conducted using a two step procedure by first estimating individual networks and then combining them with an edge wise criterion. Although this procedure works well for our application, it potentially introduces bias in estimating the group level network by ignoring the variability of the 
individual networks. One may explore the utility of developing a different network aggregation rule that can incorporate the individual network variability measurements, which may yield improved group level inferences. Also, the aggregation rule can be extended by taking into account the topology of the network instead of only considering the edges.

\section{Acknowledgement}

The authors are grateful to the support from the NIH grant: 1R01MH105561 (Jian Kang), U18 NS082143 (Dubois Bowman), R01MH073719 (Helen Mayberg), P50MH077083 (Helen Mayberg) and 1R01MH080880 (Helen Mayberg). Han Liu is grateful for the support of NSF CAREER Award DMS1454377, NSF IIS1408910, NSF IIS1332109, NIH R01MH102339, NIH R01GM083084, and NIH R01HG06841.

\section{References}

Abercrombie, H. C., Schaefer, S. M., Larson, C. L., Oakes, T. R., Lindgren, K. A., Holden, J. E., Perlman, S. B., Turski, P. A., Krahn, D. D., Benca, R. M., et al. (1998), "Metabolic rate in the right amygdala predicts negative affect in depressed patients," Neuroreport, 9, 3301-3307.

Baxter, L. R., Schwartz, J. M., Phelps, M. E., Mazziotta, J. C., Guze, B. H., Selin, C. E., Gerner, R. H., and Sumida, R. M. (1989), "Reduction of prefrontal cortex glucose metabolism common to three types of depression," Archives of general psychiatry, 46, 243-250.

Benjamini, Y. and Yekutieli, D. (2001), "The control of the false discovery rate in multiple testing under dependency," Annals of statistics, 1165-1188. 
Buckner, R. L., Andrews-Hanna, J. R., and Schacter, D. L. (2008), “The brain's default network," Annals of the New York Academy of Sciences, 1124, 1-38.

Calhoun, V., Adali, T., Pekar, J., Pearlson, G., et al. (2003), "Latency (in) sensitive ICA-Group independent component analysis of fMRI data in the temporal frequency domain," NeuroImage, 20, 1661-1669.

Craddock, R. C., Holtzheimer, P. E., Hu, X. P., and Mayberg, H. S. (2009), "Disease state prediction from resting state functional connectivity," Magnetic resonance in Medicine, 62, 1619-1628.

Drevets, W. C. (1999), "Prefrontal Cortical-Amygdalar Metabolism in Major Depression," Annals of the New York Academy of Sciences, 877, 614-637.

— (2000), "Neuroimaging studies of mood disorders," Biological psychiatry, 48, 813829.

Dunlop, B. W., Binder, E. B., Cubells, J. F., Goodman, M. G., Kelley, M. E., Kinkead, B., Kutner, M., Nemeroff, C. B., Newport, D. J., Owens, M. J., et al. (2012), "Predictors of remission in depression to individual and combined treatments (PReDICT): study protocol for a randomized controlled trial," Trials, 13, 106.

Friman, O., Cedefamn, J., Lundberg, P., Borga, M., and Knutsson, H. (2001), "Detection of neural activity in functional MRI using canonical correlation analysis," Magnetic Resonance in Medicine, 45, 323-330.

Fukumizu, K., Bach, F. R., and Gretton, A. (2007), "Statistical consistency of kernel canonical correlation analysis," The Journal of Machine Learning Research, 8, 361383. 
Guo, Y. (2011), “A General Probabilistic Model for Group Independent Component Analysis and Its Estimation Methods," Biometrics, 67, 1532-1542.

James, G. A., Kelley, M. E., Craddock, R. C., Holtzheimer, P. E., Dunlop, B. W., Nemeroff, C. B., Mayberg, H. S., and Hu, X. P. (2009), "Exploratory structural equation modeling of resting-state fMRI: applicability of group models to individual subjects," Neuroimage, 45, 778-787.

Katenka, N. and Kolaczyk, E. (2011), "Multi-Attribute Networks and the Impact of Partial Information on Inference and Characterization," Arxiv preprint $\operatorname{arXiv:1109.3160.}$

Kessler, R. C., Berglund, P., Demler, O., Jin, R., Koretz, D., Merikangas, K. R., Rush, A. J., Walters, E. E., and Wang, P. S. (2003), "The epidemiology of major depressive disorder: results from the National Comorbidity Survey Replication (NCS-R)," Jama, 289, 3095-3105.

Kuss, M. and Graepel, T. (2003), "The geometry of kernel canonical correlation analysis," Tech. rep., MPI-Technical Reports. URL http://www. kyb. mpg. de/publication. html.

Leurgans, S. E., Moyeed, R. A., and Silverman, B. W. (1993), "Canonical correlation analysis when the data are curves," Journal of the Royal Statistical Society. Series $B$ (Methodological), 725-740.

Mayberg, H. (1997), "Limbic-cortical dysregulation," The Neuropsychiatry of Limbic and Subcortical Disorders. American Psychiatric Press: Washington, DC, 167-178.

Mayberg, H., Liotti, M., Brannan, S., McGinnis, S., Mahurin, R., Jerabek, P., Silva, J., Tekell, J., Martin, C., Lancaster, J., et al. (1999), "Reciprocal limbic-cortical 
function and negative mood: converging PET findings in depression and normal sadness," American Journal of Psychiatry, 156, 675-682.

Mayberg, H., Lozano, A., Voon, V., McNeely, H., Seminowicz, D., Hamani, C., Schwalb, J., and Kennedy, S. (2005), "Deep brain stimulation for treatmentresistant depression," Neuron, 45, 651-660.

Mayberg, H. S. (2003a), "Modulating dysfunctional limbic-cortical circuits in depression: towards development of brain-based algorithms for diagnosis and optimised treatment," British medical bulletin, 65, 193-207.

— (2003b), "Positron emission tomography imaging in depression: a neural systems perspective," Neuroimaging Clinics of North America, 13, 805-815.

Nandy, R. and Cordes, D. (2004), "Improving the spatial specificity of canonical correlation analysis in fMRI," Magnetic Resonance in Medicine, 52, 947-952.

Nielsen, F. A., Hansen, L. K., Strother, S. C., Paus, T., Gjedde, A., and Evans, A. (1998), "Canonical ridge analysis with ridge parameter optimization," in 4th International Conference on Functional Mapping of the Human Brain.

Ochsner, K. N., Bunge, S. A., Gross, J. J., and Gabrieli, J. D. (2002), "Rethinking feelings: an FMRI study of the cognitive regulation of emotion," Journal of cognitive neuroscience, 14, 1215-1229.

O‘Reardon, J. P., Solvason, H. B., Janicak, P. G., Sampson, S., Isenberg, K. E., Nahas, Z., McDonald, W. M., Avery, D., Fitzgerald, P. B., Loo, C., et al. (2007), "Efficacy and safety of transcranial magnetic stimulation in the acute treatment of major depression: a multisite randomized controlled trial," Biological psychiatry, $62,1208-1216$. 
Seminowicz, D., Mayberg, H., McIntosh, A., Goldapple, K., Kennedy, S., Segal, Z., and Rafi-Tari, S. (2004), "Limbic-frontal circuitry in major depression: a path modeling metanalysis," Neuroimage, 22, 409-418.

Sheline, Y. I., Barch, D. M., Donnelly, J. M., Ollinger, J. M., Snyder, A. Z., and Mintun, M. A. (2001), "Increased amygdala response to masked emotional faces in depressed subjects resolves with antidepressant treatment: an fMRI study," Biological psychiatry, 50, 651-658.

Siegle, G. J., Steinhauer, S. R., Thase, M. E., Stenger, V. A., and Carter, C. S. (2002), "Cant shake that feeling: event-related fMRI assessment of sustained amygdala activity in response to emotional information in depressed individuals," Biological psychiatry, 51, 693-707.

Siegle, G. J., Thompson, W., Carter, C. S., Steinhauer, S. R., and Thase, M. E. (2007), "Increased amygdala and decreased dorsolateral prefrontal BOLD responses in unipolar depression: related and independent features," Biological psychiatry, 61, $198-209$

Sun, F., Miller, L., D’Esposito, M., et al. (2004), "Measuring interregional functional connectivity using coherence and partial coherence analyses of fMRI data," Neuroimage, 21, 647-658.

Supekar, K., Menon, V., Rubin, D., Musen, M., and Greicius, M. (2008), "Network analysis of intrinsic functional brain connectivity in Alzheimer's disease," PLoS computational biology, 4, e1000100.

Suykens, J. A. and Vandewalle, J. (1999), "Least squares support vector machine classifiers," Neural processing letters, 9, 293-300. 
Tzourio-Mazoyer, N., Landeau, B., Papathanassiou, D., Crivello, F., Etard, O., Delcroix, N., Mazoyer, B., and Joliot, M. (2002), "Automated anatomical labeling of activations in SPM using a macroscopic anatomical parcellation of the MNI MRI single-subject brain," Neuroimage, 15, 273-289. 


\section{Appendix}

\begin{tabular}{|c|c|c|c|c|c|}
\hline Full.Name & Short Name & Full.Name & Short Name & Full.Name & Short Name \\
\hline Precental gyrus, left & PreCG.L & Anterior cingulate and paracingulate gyri, left & ACG.L & Inferior parietal, but supramarginal and angualr gyri, left & IPL.L \\
\hline Precental gyrus, right & PreCG.R & Anterior cingulate and paracingulate gyri, right & ACG.R & Inferior parietal, but supramarginal and angualr gyri, right & IPL.R \\
\hline Superior frontal gyrus, dorsolateral, left & SFGdor.L & Median cingulate and paracingulate gyri, left & DCG.L & Supramarginal gyrus, left & SMG.L \\
\hline Superior frontal gyrus, dorsolateral, right & SFGdor.R & Median cingulate and paracingulate gyri, right & DCG.R & Supramarginal gyrus, right & SMG.R \\
\hline Superior frontal gyrus, obital part, left & ORBsup.L & Posterior cingulate grus, left & PCG.L & Angular gyrus, left & ANG.L \\
\hline Superior frontal gyrus, obital part, right & ORBsup.R & Posterior cingulate grus, right & PCG.R & Angular gyrus, right & ANG.R \\
\hline Middle frontal gyrus, left & MFG.L & Hippocampus, left & HIP.L & Precuneus, left & PCUN.L \\
\hline Middle frontal gyrus, right & MFG.R & Hippocampus, right & HIP.R & Precuneus, right & PCUN.R \\
\hline Middle frontal gyrus, orbital part, left & ORBmid.L & Parahippocampal gyrus, left & PHG.L & Paracentral lobule, left & PCL.L \\
\hline Middle frontal gyrus, orbital part, right & ORBmid.R & Parahippocampal gyrus, right & PHG.R & Paracentral lobule, right & PCL.R \\
\hline Inferior frontal gyrus, opercular part, left & IFGoperc.L & Amygdala, left & AMYG.L & Caudate nucleus, left & CAU.L \\
\hline Inferior frontal gyrus, opercular part, right & IFGoperc.R & Amygdala, right & AMYG.R & Caudate nucleus, right & CAU.R \\
\hline Inferior frontal gyrus, triangular part, left & IFGtriang.L & Calcarine fissure and surrounding cortex, left & CALLL & Lenticular nucleus, putamen, left & PUT.L \\
\hline Inferior frontal gyrus, triangular part, right & IFGtriang.R & Calcarine fissure and surrounding cortex, right & CALLR & Lenticular nucleus, putamen, right & PUT.R \\
\hline Inferior frontal gvrus, orbital part, left & ORBinf.L & Cuneus, left & CUN.L & Lenticular nucleus, pallidum, left & PALLL \\
\hline Inferior frontal gyrus, orbital part, right & ORBinf.R & Cuneus, right & CUN.R & Lenticular nucleus, pallidum, right & PAL.R \\
\hline Rolandic operculum, left & ROLLL & Lingual gyrus, left & LING.L & Thalamus, left & THA.L \\
\hline Rolandic operculum, right & ROL.R & Lingual gyrus, right & LING.R & Thalamus, right & THA.R \\
\hline Supplementary motor area, left & SMA.L & Superior occipital gyrus, left & SOG.L & Heschl gyrus, left & HES.L \\
\hline Supplementary motor area, right & SMA.R & Superior occipital gyrus, right & SOG.R & Heschl gyrus, right & HES.R \\
\hline Olfactory cortex, left & OLF.L & Middle occipital gyrus, left & MOG.L & Superior temporal gyrus, left & STG.L \\
\hline Olfactory cortex, right & OLF.R & Middle occipital gyrus, right & MOG.R & Superior temporal gyrus, right & STG.R \\
\hline Superior frantal gyrus, medial, left & SFGmed.L & Inferior occipital gyrus, left & 10G.L & Temporal pole: superior temporal gyrus, left & TPOsup.L \\
\hline Superior frantal gyrus, medial, right & SFGmed.R & Inferior occipital gyrus, right & IOG.R & Temporal pole: superior temporal gyrus, right & TPOsup.R \\
\hline Superior frontal gyrus, medial orbital, left & ORBsupmed.L & Fusiform gyrus, left & FFG.L & Middle temporal gyrus, left & MTG.L \\
\hline Superior frontal gyrus, medial orbital, right & ORBsupmed.R & Fusiform gyrus, right & FFG.R & Middle temporal gyrus, right & MTG.R \\
\hline Gyrus rectus, left & REC.L & Postcentral gyrus, left & POCG.L & Temporal pole: middle temporal gyrus, left & TPOmid.L \\
\hline Gyrus rectus, right & REC.R & Postcentral gyrus, right & POCG.R & Temporal pole: middle temporal gyrus, right & TPOmid.R \\
\hline Insula, left & INS.L & Superior parietal gyrus, left & SPG.L & Inferior temporal gyrus, left & ITG.L \\
\hline Insula, right & INS.R & Superior parietal gyrus, right & SPG.R & Inferior temporal gyrus, right & ITG.R \\
\hline
\end{tabular}

Table 7: Full names and short names of 90 AAL regions used in the paper 